\title{
Transgenerational epigenetic variance for body weight in meat quails
}

\author{
J.T. Paiva ${ }^{1}$ | M.D.V. de Resende ${ }^{2,3}$ | R.T. Resende ${ }^{2}$ | H.R. de Oliveira ${ }^{1}$ (i) | H.T. Silva ${ }^{1}$ | \\ G.C. Caetano ${ }^{1}$ ｜ P.S. Lopes ${ }^{1}$ | F.F. Silva ${ }^{1}$
}

${ }^{1}$ Department of Animal Science, Universidade Federal de Viçosa, Viçosa, Minas Gerais, Brazil

${ }^{2}$ Department of Forestry, Universidade Federal de Viçosa, Viçosa, Minas Gerais, Brazil

${ }^{3}$ EMBRAPA Forestry Research, Colombo, Paraná, Brazil

\section{Correspondence}

F.F. Silva, Universidade Federal de

Viçosa, Minas Gerais, Brazil.

Email: fabyanofonseca@ufv.br

\begin{abstract}
Summary
We aimed to estimate transgenerational epigenetic variance for body weight using genealogical and phenotypic information in meat quails. Animals were individually weighted from 1 week after hatching, with weight records at 7, 14, 21, 28, 35 and 42 days of age (BW7, BW14, BW21, BW28, BW35 and BW42, respectively). Single-trait genetic analyses were performed using mixed models with random epigenetic effects. Variance components were estimated by the restricted maximum likelihood method. A grid search for values of autorecursive parameter $(\lambda)$ ranging from 0 to 0.5 was used in the variance component estimation. This parameter is directly related to the reset coefficient $(v)$ and the epigenetic coefficient of transmissibility (1-v). The epigenetic effect was only significant for BW7. Direct heritability estimates for body weight ranged in magnitude (from 0.15 to 0.26), with the highest estimate for BW7. Epigenetic heritability was 0.10 for BW7, and close to zero for the other body weights. The inclusion of the epigenetic effect in the model helped to explain the residual and non-Mendelian variability of initial body weight in meat quails.
\end{abstract}

\section{K E Y W O R D S}

Coturnix coturnix, epigenome, non-Mendelian inheritance, reset coefficient

\section{1 | INTRODUCTION}

Epigenetic is characterized by modifications in inheritance patterns not involving changes in DNA sequence (González-Recio, Toro, \& Bach, 2015; Jablonka \& Raz, 2009; Szyf, 2015). The main modifications are given by methylation of cytosine bases and changes in histone proteins, which are called epigenetic marks and may be associated with gene expression (Heard \& Martienssen, 2014; Triantaphyllopoulos, Ikonomopoulos, \& Bannister, 2016). Epigenetic variations may occur due to environmental effects in critical periods during the animal's life and may be inherited together with DNA through generations (Ibeagha-Awemu \& Zhao, 2015). Thus, the so-called transgenerational epigenetic inheritance occurs when the marks are observed in a generation of offspring that was not exposed to the inducing environmental agent (Jablonka \& Raz, 2009; Skinner, 2011; Szyf, 2015).

Relevant researches on poultry epigenetics (Berghof, Parmentier, \& Lammers, 2013; Feeney, Nilsson, \& Skinner, 2014; Frésard et al., 2013; Gao et al., 2017; Leurox et al., 2017; Li, Guo, Zhang, Gao, \& Guo, 2015; Li et al., 2016) revealed that epigenetic modifications may occur from the first stage of egg, when the mother provides an environmental signature through the egg content (Frésard et al., 2013). However, studies approaching the transgenerational epigenetic variance estimation (Jablonka, 2013; Jablonka \& Raz, 2009; Lopes, Bastiaansen, Janss, Knol, \& Bovenhuis, 2015; Varona et al., 2015) for economically important traits are still scarce in the literature.

To the best of our knowledge, there are no reports of epigenetic variability in quails. Thus, this study may help 
to better explain the residual and non-Mendelian variability of production traits in this specie. In summary, we aimed to estimate transgenerational epigenetic variance for body weights in meat quails using genealogical and phenotypic information based on a mixed model framework. The obtained results may be useful to exploit transgenerational epigenetic heritabilities for breeding programme purposes.

\section{2 | MATERIAL AND METHODS}

\subsection{Population structure and phenotypes}

All animal procedures were approved by the Animal Care and Use Committee of the Animal Science Department from Universidade Federal de Viçosa (UFV, Brazil) (No. 73/2014-CEUAP). Data used here were collected from an experiment developed in 2014 and supported by the Breeding Program for Meat Quails of UFV. Individual observations of body weight for animals from UFV1 line (Coturnix coturnix) were used here. A total of 102 sires and 204 dams were matted under a rate of two females per each male by generation, and the proportion of sexes in the progeny was $54 \%$ female and $46 \%$ male. Eggs were collected during 10 days in each phase, incubated for 14 days and allocated in a hatcher for 3 days up to hatching. On the hatching day, progenies received identification for pedigree information control (used to form the relationship matrix). The relationship matrix was composed of 5,832 animals, up to three generations.

Animals were distributed in a total of 24 brickwork and screen boxes $(1.0 \mathrm{~m} \times 0.8 \mathrm{~m})$ used for performance evaluation. Each box had wood shavings bed, heater, dish-type feeder and pressure cup drinker. Feed and water were provided ad libitum. From the 14th day after hatch, tubular feeders and automatic nipple drinkers were used. Diet was formulated according to nutritional recommendations outlined by NRC (1994). Up to the 14th day of birds' life, lighting programme of $24 \mathrm{hr}$ of light was set, and after this period, natural lighting was used. Average offspring per couple of birds was 6.59 (standard deviation of 3.58), in which the minimum and maximum number of animals per couple were 1 and 20, respectively. Thus, a total of 2.995 offspring were evaluated. Individual weights were obtained from 1 week after hatching, with records at $7,14,21,28,35$ and 42 days of age (BW7, BW14, BW21, BW28, BW35 and BW42), being the last one considered as slaughter weight. The data collected showed differences in the total $N$ (Table 1) between the weights measurements, as there was mortality of some animals or errors during data collection, which were eliminated as potential outliers. At 21 days of age, the animals were sexed by visual identification, and at 28 days of age, the birds were the selected, adopting as selection criteria the body weight at that age. Thus, males and females presenting
T A B LE 1 Descriptive statistics of body weight (BW) at 7, 14, $21,28,35$ and 42 days of age in meat quails

\begin{tabular}{llrrrrc} 
Traits & $\boldsymbol{N}$ & $\boldsymbol{M} \pm \boldsymbol{S} \boldsymbol{S}$ & $\boldsymbol{S} \boldsymbol{D}$ & $\mathrm{CV}$ & \multicolumn{1}{c}{ MIN } & \multicolumn{1}{c}{ MAX } \\
\hline BW7 & 2.995 & $29.77 \pm 0.09$ & 4.96 & 16.67 & 11.30 & 56.70 \\
BW14 & 2.983 & $76.18 \pm 0.18$ & 9.84 & 12.92 & 31.70 & 110.20 \\
\hline BW21 & 2.987 & $134.46 \pm 0.28$ & 15.78 & 11.73 & 48.80 & 228.85 \\
\hline BW28 & 2.985 & $193.66 \pm 0.36$ & 19.97 & 10.31 & 91.56 & 297.60 \\
\hline BW35 & 2.932 & $232.22 \pm 0.40$ & 21.77 & 9.37 & 114.41 & 339.50 \\
BW42 & 2.904 & $254.77 \pm 0.50$ & 26.98 & 10.59 & 139.29 & 361.40
\end{tabular}

$N$, number of observations; $M \pm S E$, mean \pm standard error; $S D$, standard deviation; $\mathrm{CV}$, coefficient of variation; MIN, minimum value; MAX, maximum value.

highest body weights were selected to become parents of the next generation. These selected animals were posteriorly transferred to galvanized cages. These cages had dimensions of $0.90 \times 0.44 \times 0.23 \mathrm{~m}$ (length $\times$ width $\times$ height), and were equipped with linear feeders and linear drinkers between cages, supplied with running water. Animals that were not selected were maintained for body weight measurements for subsequent days until the slaughter.

\section{2 | Statistical analyses}

The animal model regarding epigenetic variation is given by:

$$
\mathbf{y}=\mathbf{X b}+\mathbf{Z} \mathbf{a}+\mathbf{Z} \xi+\mathbf{e},
$$

in which $\mathbf{y}$ is the trait vector; $\mathbf{b}$ is the vector of fixed effects; $\mathbf{a}$ is the vector of direct additive genetic effect, NID $\left(0, \sigma_{a}^{2} \mathbf{A}\right) ; \xi$ is the vector of epigenetic effects, NID $\left(0, \sigma_{\xi}^{2} \mathbf{\Lambda}\right) ; \mathbf{X}$ is the incidence matrix of fixed effects; $\mathbf{Z}$ is the incidence matrix for the direct additive genetic and epigenetic effects; and $\mathbf{e}$ is the vector of residual effects, NID $\left(0, \sigma_{e}^{2} \mathbf{I}\right)$. The considered fixed effects for all traits were the contemporary groups based on generation, hatching, year of birth and sex. The $\mathbf{A}, \boldsymbol{\Lambda}$ and $\mathbf{I}$ are the traditional additive relationship, the epigenetic relationship and the identity matrices, related respectively to the additive genetic $\left(\sigma_{a}^{2}\right)$, transgenerational epigenetic $\left(\sigma_{\xi}^{2}\right)$ and residual $\left(\sigma_{e}^{2}\right)$ variances. Variance components and genetic parameters were estimated by the restricted maximum likelihood method (REML) using the package regress of $\mathrm{R}$ software (R Core Team, 2017), which uses the Newton-Raphson algorithm to identify the maximum of the log-likelihood surface.

Given the assumption of independence between $\mathbf{a}$ and $\xi$, and a possible genetic covariance between them, we evaluated $\hat{\xi}$ in function of $\hat{\mathbf{a}}$ by fitting a linear regression model under a Bayesian framework. Thus, the hypothesis of null slope was exploited through posterior credible intervals (CI). If the 95\% CI contains the value zero, the assumption of independence between the effects can be 
confirmed. The Bayesian regression analysis was implemented by MCMCregress function of $\mathrm{R}$ software considering a total of 100,000 iterations with a burn-in period and sampling interval (thin) of 50,000 and five iterations, respectively.

The structure of the $\boldsymbol{\Lambda}$ matrix is defined by the recursive relationship between the epigenetic effect of one individual $\left(\xi_{i}\right)$ with respect to the epigenetic effects of its father $\left(\xi_{p i}\right)$ and mother $\left(\xi_{m i}\right)$ (Varona et al., 2015):

$$
\xi_{i}=\lambda \xi_{p i}+\lambda \xi_{m i}+\varepsilon_{i}
$$

The $\boldsymbol{\Lambda}$ depends particularly on a single autorecursive parameter $(\lambda)$, which is directly related to the reset coefficient $(v)$, where $\lambda=0.5(1-v)$. According to Tal, Kisdi, and Jablonka (2010), the covariance between relatives is redefined in function of transgenerational epigenetic variance, the reset coefficient $(v)$ and the epigenetic transmission coefficient $(1-v)$. The covariance between relatives is possible to distinguish between the additive genetic and the epigenetic inheritance via quantitative genetics theory. In summary, the parameter $v$ represents the proportion of epigenetic marks present in the parental genome that will be erased, whereas the $(1-v)$ is its complement and indicates the proportion of these marks that will be transmitted to progeny. The residual epigenetic effect of the $i$ th individual, independent from $\xi_{i}$, follows these distributions:

$\varepsilon_{i} \sim N\left(0,\left(1-2 \lambda^{2}\right) \sigma_{\xi}^{2}\right)$ if both parents are known;

$\varepsilon_{i} \sim N\left(0,\left(1-\lambda^{2}\right) \sigma_{\xi}^{2}\right)$ if only one ancestor is known.

Assuming that the variance of transgenerational epigenetic effects is constant across generations (Varona et al., 2015), we have:

$$
V\left(\xi_{i}\right)=V\left(\xi_{p i}\right)=V\left(\xi_{m i}\right)=\sigma_{\xi}^{2}
$$

which in matrix notation refers to:

$$
\xi=\mathbf{P} \xi+\varepsilon
$$

where $\mathbf{P}$ is the matrix that defines the recurrent relationship with the epigenetic effects of the father and mother. For non-base individuals, the row of the $i$ th individual of the $\mathbf{P}$ matrix has a parameter $\lambda$ in the column belonging to the father and mother of the $i$ th individual; and the rest of elements are null (Varona et al., 2015). Furthermore, if:

$$
\xi=[\mathbf{I}-\mathbf{P}]^{-1} \boldsymbol{\varepsilon}
$$

then

$$
\mathbf{V}(\boldsymbol{\xi})=\boldsymbol{\Lambda} \boldsymbol{\sigma}_{\xi}^{2}=[\mathbf{I}-\mathbf{P}]^{-1} V(\boldsymbol{\varepsilon})\left[\mathbf{I}-\mathbf{P}^{\prime}\right]^{-1},
$$

where $\mathbf{V}(\boldsymbol{\varepsilon})$ is a diagonal matrix with entries equal to $\sigma_{\xi}^{2}$ for base individuals, $\left(1-\lambda^{2}\right) \sigma_{\xi}^{2}$ for individuals with only one known ancestor and $\left(1-2 \lambda^{2}\right) \sigma_{\xi}^{2}$ for individuals whose father and mother are known. Thereby, the epigenetic heritability can be calculated as $h_{\xi}^{2}=\frac{\sigma_{\xi}^{2}}{\sigma^{2}}$. In summary, the model used can be fitted through the mixed model equations as follow:

$$
\left[\begin{array}{ccc}
\mathbf{X}^{\prime} \mathbf{X} & \mathbf{X}^{\prime} \mathbf{Z} & \mathbf{X}^{\prime} \mathbf{Z} \\
\mathbf{Z}^{\prime} \mathbf{X} & \mathbf{Z}^{\prime} \mathbf{Z}+\mathbf{A}^{-1} \frac{\boldsymbol{\sigma}_{e}^{2}}{\boldsymbol{\sigma}_{g}^{2}} & \mathbf{Z}^{\prime} \mathbf{Z} \\
\mathbf{Z}^{\prime} \mathbf{X} & \mathbf{Z}^{\prime} \mathbf{Z} & \mathbf{Z}^{\prime} \mathbf{Z}+\boldsymbol{\Lambda}^{-1} \frac{\boldsymbol{\sigma}_{e}^{2}}{\boldsymbol{\sigma}_{\xi}^{2}}
\end{array}\right]\left[\begin{array}{c}
\hat{\mathbf{b}} \\
\hat{\mathbf{g}} \\
\hat{\xi}
\end{array}\right]=\left[\begin{array}{c}
\mathbf{X}^{\prime} \mathbf{y} \\
\mathbf{Z}^{\prime} \mathbf{y} \\
\mathbf{Z}^{\prime} \mathbf{y}
\end{array}\right]
$$

which requires the inverse of the $\mathbf{A}$ and $\boldsymbol{\Lambda}$ matrices. The inverse of the A matrix was obtained as in the standard model of Henderson (1984), and the inverse of the $\boldsymbol{\Lambda}$ matrix was obtained as described by Varona et al. (2015). The procedure to invert the $\boldsymbol{\Lambda}$ matrix takes into account the recursive nature of the transgenerational epigenetic effects, using an argument similar to Quass (1976) for the inverse of $\mathbf{A}$. The algorithm for the construction of $\boldsymbol{\Lambda}^{-1}$ matrix can be described as (Varona et al., 2015):

i If both father and mother are unknown: add 1 to the diagonal (i, i);

ii If only one parent (p) is known: add $\frac{1}{\left(1-\lambda^{2}\right)}$ to the diagonal (i, i), add $\frac{-\lambda}{\left(1-\lambda^{2}\right)}$ to the elements (i, p), (p,i) and add $\frac{\lambda^{2}}{\left(1-\lambda^{2}\right)}$ to the element $(\mathrm{p}, \mathrm{p})$; and

iii If both ancestors ( $\mathrm{p}$ and $\mathrm{m}$ ) are known: add $\frac{1}{\left(1-2 \lambda^{2}\right)}$ to the diagonal (i, i), add $\frac{-\lambda}{\left(1-2 \lambda^{2}\right)}$ to the elements (i, p), (i, m), (m, i), (p, i) and add $\frac{\lambda^{2}}{\left(1-2 \lambda^{2}\right)}$ to the elements (p, $\mathrm{p}),(\mathrm{p}, \mathrm{m}),(\mathrm{m}, \mathrm{p}),(\mathrm{m}, \mathrm{m})$.

Based on this algorithm, we developed a script in the $\mathrm{R}$ software for the construction of the inverse of $\boldsymbol{\Lambda}$ (available at Appendix S1). A grid search considering values of $\lambda$ ranging from 0 to 0.5 , with interval of 0.01 , were used to estimate the variance components. Based on the Akaike information criterion (AIC), the best $\lambda$ value was chosen to be used in the previously mentioned formulas.

Analyses were also performed using the reduced model (without the epigenetic effect) through the "regress" package of R software (2016). To compare the significance of the difference in the fit between the reduced and epigenetic models, the likelihood ratio test (LRT) was performed. The breeding values of the individuals were predicted for each trait using both models (reduced and with epigenetic effect), and the Spearman correlations were calculated to quantify the degree of concordance between them.

\section{3 | RESULTS}

The descriptive statistics of body weight at different ages are presented in Table 1.

For the majority of the body weights (BW14, BW21 and BW35), the $\lambda$ value was 0.50 , whereas for BW7, 
BW42 and BW28, this value decreased to $0.39,0.22$ and 0.05 , respectively (Table 2). As this autorecursive parameter is associated with the epigenetic transmission coefficient (Tal et al., 2010), it was possible to find out the reset coefficient $(v)$ and the epigenetic transmission coefficient $(1-v)$ for each evaluated trait.

The posterior distribution for the slope of the regression of epigenetic effects in function of additive genetic effects is presented in Figure 1. We verified that the 95\% credible intervals contain the value zero, thus indicating the independence between these effects for all evaluated traits.

Table 2 shows the additive, epigenetic, residual and phenotypic variance components, and the direct and epigenetic heritability estimates for each trait obtained from both epigenetic and reduced (only additive genetic) models. According to the LRT, the epigenetic model outperformed the reduced model only for BW7.

The estimates of direct heritability for body weights over time showed variation in their magnitude considering both models. The BW7 presented moderate heritability estimates of 0.26 and 0.18 for the epigenetic and reduced models, respectively. Throughout the animal's life, the heritability estimates for other body weights (BW14, BW21 and BW28) did not vary substantially (0.15-0.17). For BW35 and BW42, the heritability estimates were greater, ranging from 0.20 to 0.22 . The transgenerational epigenetic heritability estimates for body weights were low, being the highest value obtained for BW7 (0.10). For the other body weights, these estimates were low or close to zero.

The breeding values predicted by the reduced model showed high correlation with those predicted by the epigenetic model. A correlation of 0.96 was reported for BW14, whereas correlations close to 0.99 were observed for the other body weights.

\section{4 | DISCUSSION}

In terms of the assumed independence between epigenetic and additive genetic effects, as the slope was statistically null (95\% credible interval contains the value zero) for all studied traits (Figure 1), we believe that the covariance between these effects would really be really fixed at zero. However, more sophisticated models assuming covariance between these effects would be fitted in future analysis to test the independence based on unknown measurements obtained for the covariance estimates.

TA B LE 2 Estimates of variance components (with respective standard errors), genetic and epigenetic parameters (Par) for body weight in meat quails using epigenetic (I) and additive genetic (II) models

\begin{tabular}{|c|c|c|c|c|c|c|c|}
\hline Model & Par & BW7 & BW14 & BW21 & BW28 & BW35 & BW42 \\
\hline \multirow[t]{8}{*}{ I } & $\sigma_{a}^{2}$ & $3.42 \pm 0.87$ & $12.44 \pm 2.62$ & $29.06 \pm 4,75$ & $54.065 \pm 16.36$ & $75.12 \pm 10.44$ & $96.40 \pm 26.52$ \\
\hline & $\sigma_{\xi}^{2}$ & $1.40 \pm 0.37$ & $0.68 \pm 0.44$ & $1.93 \pm 1.16$ & $2.5 \mathrm{E}-08 \pm 114.60$ & $0.63 \pm 1.84$ & $22.51 \pm 20.25$ \\
\hline & $\sigma_{p}^{2}$ & 12.91 & 69.44 & 176.44 & 306.66 & 348.94 & 437.41 \\
\hline & $h^{2}$ & $0.26 \pm 0.03$ & $0.17 \pm 0.02$ & $0.16 \pm 0.02$ & $0.17 \pm 0.02$ & $0.21 \pm 0.03$ & $0.22 \pm 0.03$ \\
\hline & $\lambda$ & 0.39 & 0.50 & 0.50 & 0.05 & 0.50 & 0.22 \\
\hline & $v$ & 0.22 & 0.00 & 0.00 & 0.90 & 0.00 & 0.56 \\
\hline & $1-v$ & 0.78 & 1.00 & 1.00 & 0.10 & 1.00 & 0.44 \\
\hline & $\log L$ & $-5,565.8$ & $-7,931.6$ & $-9,325.6$ & $-10,110.6$ & $-10,139.8$ & $-10,384.0$ \\
\hline \multirow{5}{*}{ II } & $\sigma_{p}^{2}$ & 14.26 & 71.30 & 181.72 & 306.64 & 350.82 & 445.23 \\
\hline & $h^{2}$ & $0.18 \pm 0.01$ & $0.16 \pm 0.01$ & $0.15 \pm 0.01$ & $0.17 \pm 0.01$ & $0.21 \pm 0.01$ & $0.20 \pm 0.01$ \\
\hline & $\log L$ & $-5,574.4$ & $-7,932.5$ & $-9,327.3$ & $-10,110.6$ & $-10,139.9$ & $-10,385.0$ \\
\hline & AIC & $11,152.9$ & $15,869.1$ & $18,658.6$ & $20,225.2$ & $20,283.8$ & $20,774.0$ \\
\hline & LRT & $17.2^{\mathrm{a}}$ & $1.9^{\mathrm{ns}}$ & $3.4^{\mathrm{ns}}$ & $0.0^{\mathrm{ns}}$ & $0.2^{\mathrm{ns}}$ & $1.2^{\mathrm{ns}}$ \\
\hline
\end{tabular}

$\sigma_{a}^{2}$, additive genetic variance; $\sigma_{\xi}^{2}$, transgenerational epigenetic variance; $\sigma_{e}^{2}$, residual variance; $\sigma_{p}^{2}$, phenotypic variance; $h^{2}$, additive heritability \pm standard error; $\gamma^{2}$, transgenerational epigenetic heritability \pm standard error; $\lambda$, autorecursive parameter; $v$, reset coefficient; $1-v$, epigenetic transmission coefficient; AIC, Akaike information criterion; LogL, natural logarithm of the likelihood function; LRT, likelihood ratio test ('Significance level at 5\%; ns, no significant). 

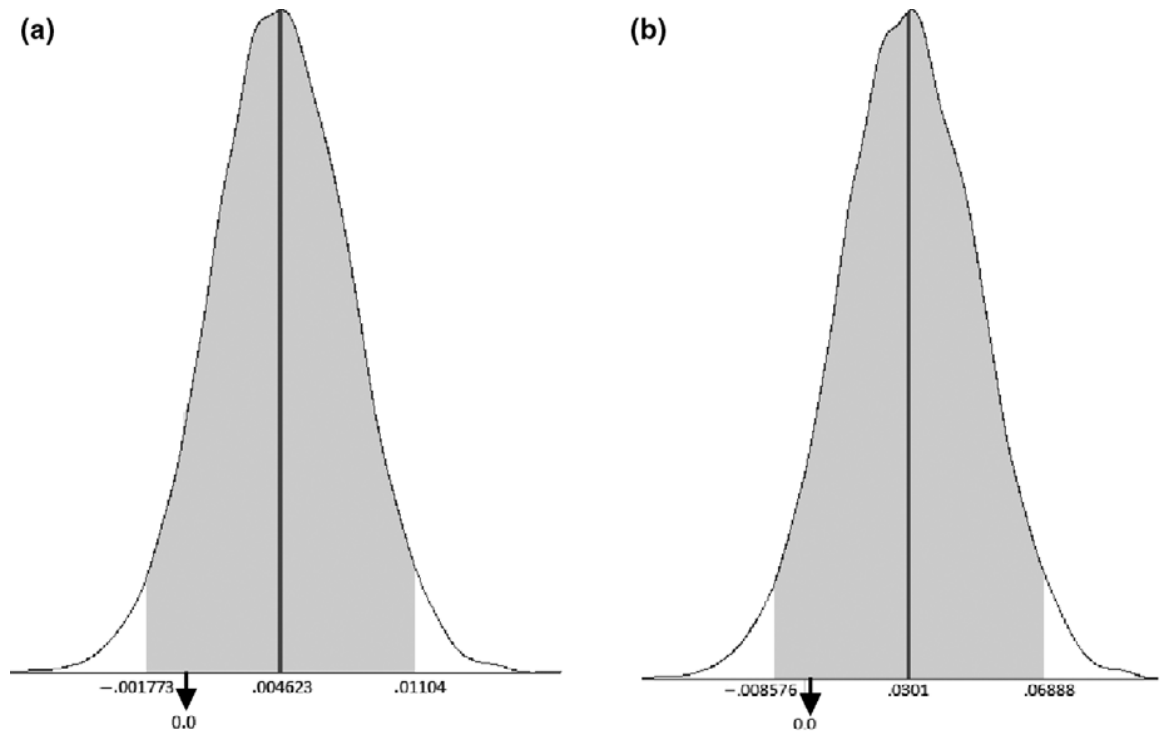

(c)

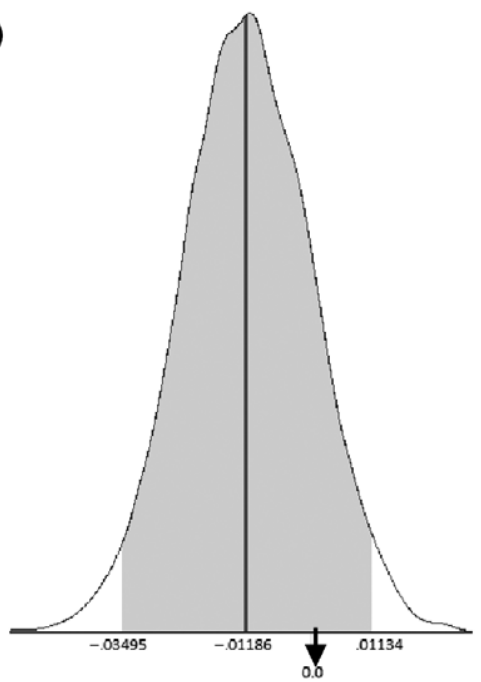

(d)
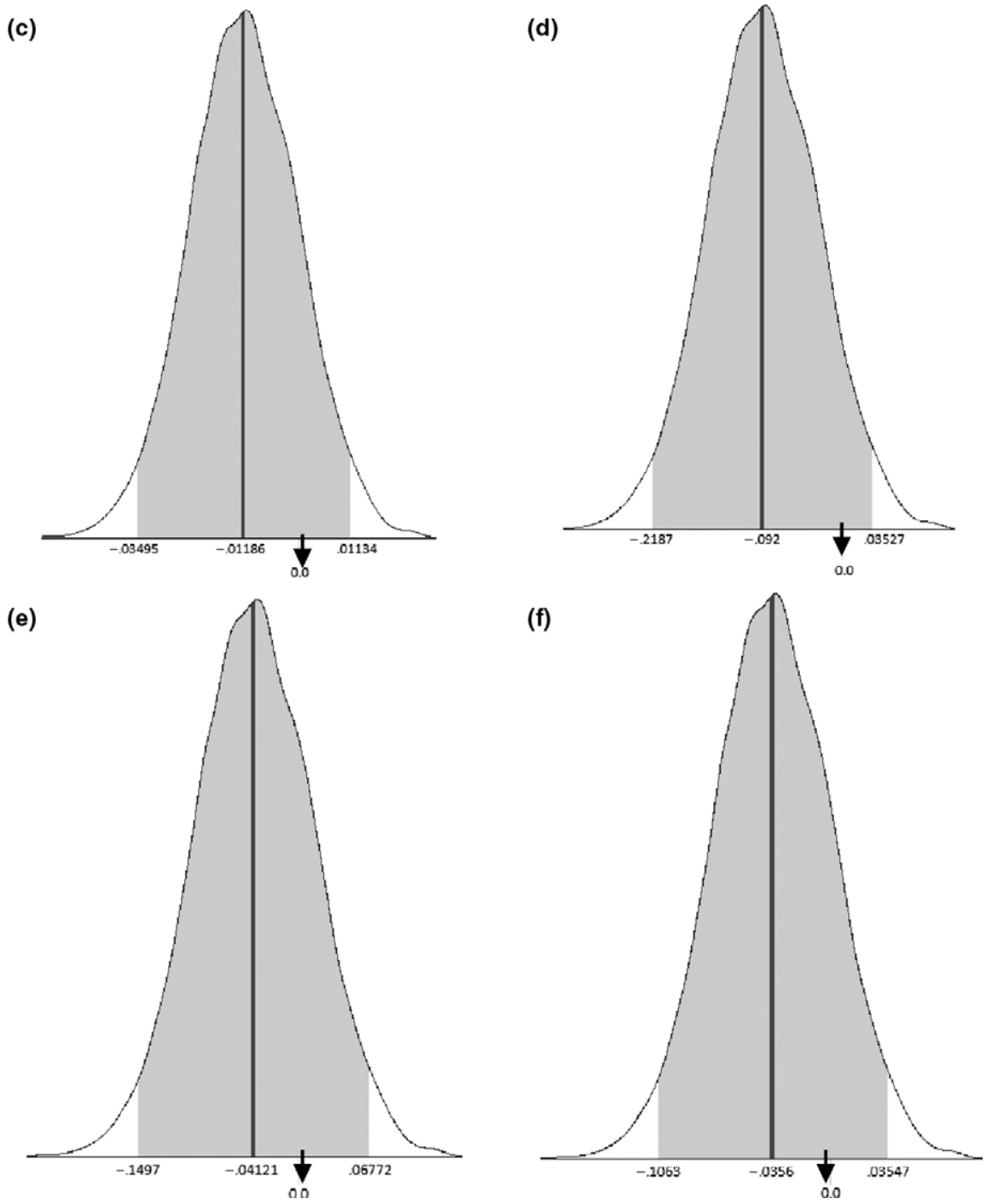

F IGURE 1 Posterior distributions for slopes from regression between epigenetic and additive genetic effects with respective $95 \%$ credible intervals for the traits BW7 (a), BW14 (b), BW21 (c), BW28 (d), BW35 (e) and BW42 (f). 
When the epigenetic component involved in the expression of body weight was evaluated, we observed that weight at 7 days of age presented transgenerational epigenetic variation, with epigenetic heritability of 0.10 . From this period, the body weight showed little or almost no epigenetic variance response on this trait, with epigenetic heritabilities close to zero for all subsequent body weights evaluated over the animals' life. Thus, it is suggested that epigenetic marks were inherited for body weight in the first week of life, and that the extension of phenotypic variation of this trait can be explained by epigenetic changes that occurred, possibly, during the process of egg formation. In this sense, such epigenetic changes would have been better evaluated by body weight assessment at hatching. Thus, subsequent studies can be carried out to evaluate the influence of epigenetic marks on birthweight in quails. Since, for birds, maternal nutritional modulation ends once the eggs are laid (Moraes et al., 2014), it is suggested that from this moment, there is a greater environmental influence on the growth traits, as reported through heritability estimates found for the animals studied (Table 2). The body weight of the birds after the first week of life seems to have greater effect of nutritional management and environmental conditions than the effects coming from genetics and/or epigenetic marks.

Studies have shown that maternal nutrition can influence the growth and carcass traits of the progeny in chicken (An, Liu, Guo, \& Sun, 2012; Callini \& Sirri, 2007; Moraes et al., 2014). In birds, the maternal nutrition can directly affect offspring performance through incorporation of nutrients into the egg or triggering epigenetic modifications that regulate muscle progenitor cells (Berghof et al., 2013; Moraes et al., 2014). Studies have reported the existence of epigenetic effects in birds, and even indicate the possibility of epigenetic inheritance, although few studies evaluate the DNA methylation or the histone modification (Berghof et al., 2013; Feeney et al., 2014; Gao et al., 2017; Li et al., 2016). Nätt et al. (2009) suggested the existence of epigenetic effects in birds evaluating two groups of hens with different light rhythms. Birds with unpredictable light rhythm showed different foraging behaviour and the offspring of these two groups presented the same foraging behaviour of their parents, even though both groups were submitted to a normal predictable light rhythm. During the incubation process, the embryo is vulnerable to the impacts of nutrients and other external environments, which can lead to epigenome reprogramming (Gao et al., 2017). In fact, it has been reported that dynamic DNA methylation and histone acetylation are involved in the differentiation of chick embryonic germ cells (Jiao et al., 2013). Thereby, a site-specific methylation pattern or other epigenetic marks will likely participate in the regulation of DNA methylation during the embryonic development in birds.

The epigenetic programming can occur at two critical periods in birds: during the period of gametogenesis or during the egg formation, when egg nutrients affect the embryo (Ferket, 2012). The embryogenesis stage is critical for epigenetic reprogramming, and it is of great importance for birds ( $\mathrm{Li}$ et al., 2016). In fact, the perinatal period, which starts at the last 4 days of incubation and goes up to 4 days after hatching, is the most critical period of development. Thus, the perinatal period may have a relevant effect on traits such as growth and health over the animals' life. There is evidence that environmental and nutritional stimuli of breeders during the perinatal period may programme how the genes will be expressed as an adaptive response to increase the survival chances of offspring (Ferket, 2012). Any effect that influences DNA methylation patterns during development may alter an animal's response throughout life and for many generations.

Mating programmes in meat quails could be designed considering the most favourable epigenetic state to complement the breeding value. Once selection is absent, all effects achieved by previous selection on the epigenetic variability will be lost (at a rate depending on epigenetic transmissibility), unless the phenotype is stabilized by genetic assimilation (Tal et al., 2010). The practical implementation should be carefully evaluated to successfully incorporate the epigenetic information into breeding programmes of meat quails. One of the main challenges is to track the epigenetic information that changes in each successive generation at the cellular level (González-Recio, 2012). Varona et al. (2015) suggest that both additive genetic and epigenetic effects can be used to predict the future performance of the animal. However, the authors advise that new research must be done to develop adequate selection indexes that consider these effects. Although both of them affect the immediate future performance of the offspring, epigenetic effects are diluted in future generations as the epigenetic marks are erased.

In summary, the epigenetic mechanisms may be responsible for a portion of missing variation in the production traits, and, possibly, for a portion of heritability that is not accounted for in the existing genetic data (Ibeagha-Awemu \& Zhao, 2015; Slatkin, 2009).

\section{5 | CONCLUSIONS}

The epigenetic effect was significant only for body weight at 7 days of age (BW7). Direct heritability estimates for body weight ranged in magnitude (from 0.15 to 0.26 ), with the highest estimate for BW7. Epigenetic heritability was 0.10 for BW7 and close to zero for all other body weights. 
The inclusion of the epigenetic effect in the model helped to explain the residual and non-Mendelian variability of initial body weight in meat quails.

\section{ORCID}

H.R. de Oliveira (D) http://orcid.org/0000-0002-0355-8902

F.F. Silva (D) http://orcid.org/0000-0001-9536-1113

\section{REFERENCES}

An, S., Liu, G., Guo, Y., \& Sun, Q. (2012). Effects of maternal and posthatch dietary oils and vitamin $\mathrm{E}$ on antioxidant capability and muscle quality of the progeny broilers. The Journal of Poultry Science, 49, 191-195. https://doi.org/10.2141/jpsa.011108

Berghof, T. V. L., Parmentier, H. K., \& Lammers, A. (2013). Transgenerational epigenetic effects on innate immunity in broilers: An underestimated field to be explored? Poultry Science, 92(11), 2904-2913. https://doi.org/10.3382/ps.2013-03177

Callini, F., \& Sirri, F. (2007). Breeder nutrition and offspring performance. Brazilian Journal of Poultry Science, 9(2), 77-83. https://doi.org/10.1590/S1516-635X2007000200001

Feeney, A., Nilsson, E., \& Skinner, M. K. (2014). Epigenetics and transgenerational inheritance in domesticated farm animals. Journal of Animal Science and Biotechnology, 5(1), 48. https://doi.org/ 10.1186/2049-1891-5-48

Ferket, P. R. (2012). Embryo epigenomic response to breeder management and nutrition. In: Proceedings, XXIV World's Poultry Congress, Salvador, Brasil, August 2012.

Frésard, L., Morisson, M., Brun, J. M., Collin, A., Pain, B., Minvielle, F., \& Pitel, F. (2013). Epigenetics and phenotypic variability: Some interesting insights from birds. Genetics Selection Evolution, 45(1), 16. https://doi.org/10.1186/1297-9686-45-16

Gao, M. M., Zhao, L., Zhang, J. L., Li, L. J., Yu, L. L., Lv, P. A., .. Zhou, G. H. (2017). Effects of in ovo feeding of 1-arginine on the development of lymphoid organs and small intestinal immune barrier function in post hatch broilers. Animal Feed Science and Technology, 225, 8-19. https://doi.org/10.1016/j.anifeedsci.2017. 01.004

González-Recio, O. (2012). Epigenetics: A new challenge in the postgenomic era of livestock. Frontiers in Genetics, 2, 106. https://d oi.org/10.3389/fgene.2011.00106

González-Recio, O., Toro, M. A., \& Bach, A. (2015). Past, present, and future of epigenetics applied to livestock breeding. Frontiers in Genetics, 6, 305. https://doi.org/10.3389/fgene.2015.00305

Heard, E., \& Martienssen, R. A. (2014). Transgenerational epigenetic inheritance: Myths and mechanisms. Cell, 157(1), 95-109. https://doi.org/10.1016/j.cell.2014.02.045

Henderson, C. R. (1984). Application of linear models in animal breeding. Guelph, ON: University of Guelph.

Ibeagha-Awemu, E. M., \& Zhao, X. (2015). Epigenetic marks: Regulators of livestock phenotypes and conceivable sources of missing variation in livestock improvement programs. Frontiers in Genetics, 6, 302. https://doi.org/10.3389/fgene.2015.00302

Jablonka, E. (2013). Epigenetic inheritance and plasticity: The responsive germline. Progress Biophysics Molecular Biology, 111, 99107. https://doi.org/10.1016/j.pbiomolbio.2012.08.014
Jablonka, E., \& Raz, G. (2009). Transgenerational epigenetic inheritance: Prevalence, mechanisms, and implications for the study of heredity and evolution. The Quarterly Review of Biology, 84(2), 131-176. https://doi.org/10.1086/598822

Jiao, F., Wang, X., Yan, Z., Liu, C., Yue, Z., Li, Z., ... Wang, J. (2013). Effect of dynamic DNA methylation and histone acetylation on cPouV expression in differentiation of chick embryonic germ cells. Stem Cells and Development, 22, 2725-2735. https:// doi.org/10.1089/scd.2013.0046

Leurox, S., Gourichon, D., Leterrier, C., Labrune, Y., Coustham, V., Rivière, S., ... Pitel, F. (2017). Embryonic environment and transgenerational effects in quail. Genetics Selection Evolution, 49, 14. https://doi.org/10.1186/s12711-017-0292-7

Li, C., Guo, S., Zhang, M., Gao, J., \& Guo, Y. (2015). DNA methylation and histone modification patterns during the late embryonic and early postnatal development of chickens. Poultry Science, 94, 706-721. https://doi.org/10.3382/ps/pev016

Li, S., Zhu, Y., Zhi, L., Han, X., Shen, J., Liu, Y., ... Yang, X. (2016). DNA Methylation variation trends during the embryonic development of chicken. PLoS One, 11(7), https://doi.org/10.1371/ journal.pone.0159230

Lopes, M. S., Bastiaansen, J. W., Janss, L., Knol, E. F., \& Bovenhuis, H. (2015). Estimation of additive, dominance, and imprinting genetic variance using genomic data. G3: Genes|Genomes|Genetics, 5(12), 2629-2637. https://doi.org/10.1534/g3.115.019513

Moraes, T. G. V., Pishnamazi, A., Mba, E. T., Wenger, I. I., Renema, R. A., \& Zuidhof, M. J. (2014). Effect of maternal dietary energy and protein on live performance and yield dynamics of broiler progeny from young breeders. Poultry Science, 93, 2818-2826. https://doi.org/10.3382/ps.2014-03928

Nätt, D., Lindqvist, N., Stranneheim, H., Lundeberg, J., Torjesen, P. A., \& Jensen, P. (2009). Inheritance of acquired behaviour adaptations and brain gene expression in chickens. PLoS One, 4(7), e6405. https://doi.org/10.1371/journal.pone.0006405

NRC (1994). Nutrient requirements of poultry. 9th Revised ed. Washington, DC: The National Academies Press. https://doi.org/10. $17226 / 2114$

Quass, R. L. (1976). Computing the diagonal elements of the inverse of a large numerator relationship matrix. Biometrics, 32, 949-953. https://doi.org/10.2307/2529279

R Core Team (2017). R: A language and environment for statistical computing. Vienna, Austria: R Foundation for Statistical Computing. Retrieved from http://www.R-project.org.

Skinner, M. K. (2011). Environmental epigenetic transgenerational inheritance and somatic epigenetic mitotic stability. Epigenetics, 6 (7), 838-842.

Slatkin, M. (2009). Epigenetic inheritance and the missing heritability problem. Genetics, 182, 845-850. https://doi.org/10.1534/genetics. 109.102798

Szyf, M. (2015). Nongenetic inheritance and transgenerational epigenetics. Trends in Molecular Medicine, 21, 134-144. https://doi. org/10.1016/j.molmed.2014.12.004

Tal, O., Kisdi, E., \& Jablonka, E. (2010). Epigenetic contribution to covariance between relatives. Genetics, 184(4), 1037-1050. https://doi.org/10.1534/genetics.109.112466

Triantaphyllopoulos, K. A., Ikonomopoulos, I., \& Bannister, A. J. (2016). Epigenetics and inheritance of phenotype variation in livestock. Epigenetics Chromatin, 9, 31. https://doi.org/10.1186/ s13072-016-0081-5 
Varona, L., Munilla, S., Mouresan, E. F., González-Rodríguez, A., Moreno, C., \& Altarriba, J. (2015). A Bayesian model for the analysis of transgenerational epigenetic variation. G3: Genes $\mid$ Genomes| Genetics, 5(4), 477-485. https://doi.org/10.1534/g3.115.016725

\section{SUPPORTING INFORMATION}

Additional supporting information may be found online in the Supporting Information section at the end of the article.
How to cite this article: Paiva JT, de Resende MDV, Resende RT, et al. Transgenerational epigenetic variance for body weight in meat quails. $J$ Anim Breed Genet. 2018;135:178-185.

https://doi.org/10.1111/jbg.12329 